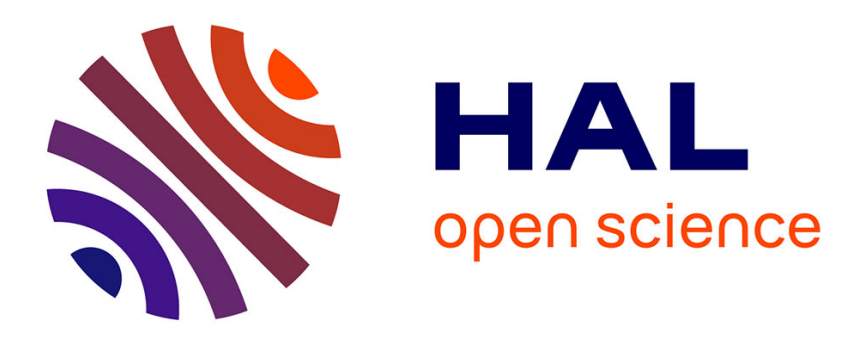

\title{
Transfert d'énergie thermique rayonnante entre deux diélectriques aux très basses températures
}

Alfredo Olivei

\section{To cite this version:}

Alfredo Olivei. Transfert d'énergie thermique rayonnante entre deux diélectriques aux très basses températures. Revue de Physique Appliquée, 1968, 3 (3), pp.225-230. 10.1051/rphysap:0196800303022500 . jpa-00242852

\section{HAL Id: jpa-00242852 https://hal.science/jpa-00242852}

Submitted on 1 Jan 1968

HAL is a multi-disciplinary open access archive for the deposit and dissemination of scientific research documents, whether they are published or not. The documents may come from teaching and research institutions in France or abroad, or from public or private research centers.
L'archive ouverte pluridisciplinaire HAL, est destinée au dépôt et à la diffusion de documents scientifiques de niveau recherche, publiés ou non, émanant des établissements d'enseignement et de recherche français ou étrangers, des laboratoires publics ou privés. 


\title{
TRANSFERT D'ÉNERGIE THERMIQUE RAYONNANTE ENTRE DEUX DIÉLEGTRIQUES AUX TRÈS BASSES TEMPÉRATURES
}

\author{
Par Alfredo OllVEI, \\ Direzione Progetti Speciali, Olivetti, Ioor5 Ivrea, Italie.
}

(Reçu le 17 avril 1968.)

\begin{abstract}
Résumé. - On montre que le transfert d'énergie thermique rayonnante entre deux diélectriques, séparés par du vide et maintenus à deux températures très basses et uniformes, est la somme de l'énergie échangée par effet tunnel et par transmission, compte tenu des effets d'interférences dues à l'espacement entre les deux diélectriques.

La solution classique ne tient pas compte de l'effet d'interférence et de l'effet tunnel.

Ces deux effets sont importants lorsque l'espacement entre les deux diélectriques est $\mathrm{du}$ même ordre de grandeur que la longueur d'onde $\lambda_{\max }$ déterminée par la loi de déplacement de Wien à une température donnée.

Abstract. - At cryogenic temperatures the energy transfer by thermal radiation between two dielectrics separated by a vacuum gap is the sum of radiation tunneling and radiation transmission, including the effect of wave interference.

The classical solution neglects the interference and the tunnel effects.

These effects have a strong influence when the surface spacing is of same order as the vacuum wavelength $\lambda_{\max }$ determined by Wien's law at a given temperature.
\end{abstract}

1. Introduction. - Dans la construction des enceintes isolées thermiquement pour le stockage aux très basses températures (de l'ordre de $2{ }^{\circ} \mathrm{K}$ ) de composés cryogéniques, les espacements entre les différentes couches isolantes qui constituent les parois de l'enceinte sont généralement du même ordre de grandeur que la longueur d'onde $\lambda_{\max }$ donnée par la relation de Wien pour le rayonnement thermique à ces très basses températures. Soient deux diélectriques I et III considérés comme étant semi-infinis et séparés par une lame de vide à faces parallèles d'épaisseur $d$.

Soient des axes $\mathrm{X}$ et $\mathrm{Y}$ définis dans le plan des

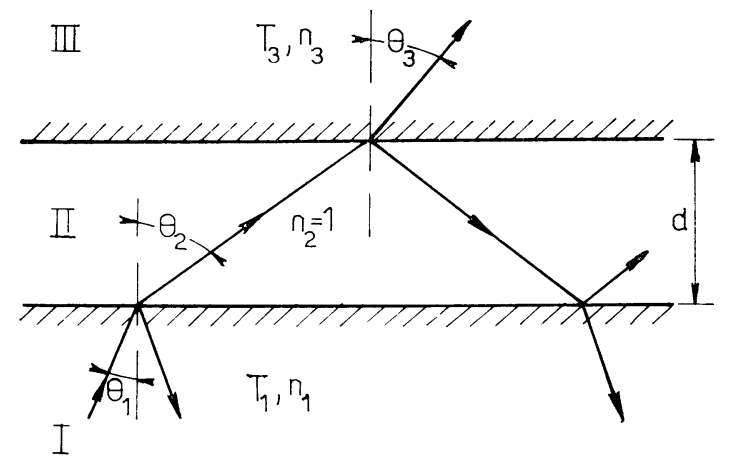

FIG. 1.

Configuration schématique du système étudié. faces et un axe $\mathrm{Z}$ perpendiculaire aux faces ( fig. 1).

Le milieu II peut être considéré comme approchant du vide, et dans tous les calculs qui suivront on prendra :

$$
n_{2}=1 \text {. }
$$

Les milieux I et III sont des diélectriques présentant des pertes faibles et ayant des constantes optiques différentes.

On va montrer que le transfert d'énergie rayonnante entre ces deux milieux diélectriques maintenus à des températures constantes et différentes est une fonction très compliquée de la distance $d$, de l'indice de réfraction des deux milieux diélectriques et de la température de ces mêmes milieux.

La solution classique ne tient pas compte de l'effet dû à la présence du milieu III sur l'angle d'incidence $\theta_{1}$ dans le milieu $I$, puisqu'elle ne considère que les angles d'incidence $\theta_{1}$ compris entre zéro et l'angle limite de réflexion totale, si bien que le possible passage d'énergie rayonnante par effet tunnel pour des angles d'incidence $\theta_{1}$ supérieurs à l'angle limite de réflexion totale est complètement négligé.

De plus, la solution classique ne tient pas compte des phénomènes d'interférence qui interviennent par suite des réflexions multiples entre la surface de séparation des milieux I et II, et la surface de séparation des milieux II et III. 
Ce phénomène d'interférence aura un effet positif ou négatif sur le transfert d'énergie selon la valeur de l'espacement $d$ et les valeurs des longueurs d'onde émises.

Pour ce qui concerne le passage d'énergie rayonnante par effet tunnel, il est bien connu que, quand une onde électromagnétique passe d'un milieu d'indice de réfraction plus élevé à un milieu d'indice moins élevé avec un angle d'incidence supérieur à l'angle limite de réflexion totale, bien qu'il y ait réflexion totale, il existe dans le deuxième milieu une onde électromagnétique évanescente dont l'amplitude décroît exponentiellement en fonction de la distance de la surface d'incidence.

La valeur moyenne du vecteur de Poynting est dans ce cas nulle. Supposons maintenant que le deuxième milieu ait une épaisseur finie et qu'il soit en contact avec un troisième milieu.

Alors, si le deuxième milieu a une épaisseur telle que le champ électromagnétique évanescent a une amplitude différente de zéro à la surface de séparation entre le deuxième et le troisième milieu, la valeur moyenne du vecteur de Poynting calculée à la surface de séparation entre le premier et le deuxième milieu sera différente de zéro : il y a donc un transfert d'énergie entre le premier et le troisième milieu.

Puisque l'énergie rayonnante thermique n'est pas polarisée, le facteur $\mathscr{J}$ de transmission entre les milieux I et III est donné par la formule suivante [1] :

$$
\mathscr{J}_{\mathrm{I} \rightarrow \mathrm{III}}=\frac{1}{2}\left(\mathscr{J}_{\perp}+\mathscr{J}_{\text {III }}\right)
$$

où $\mathscr{J}_{\perp}$ et $\mathscr{J}_{11}$ sont respectivement le facteur de transmission pour une onde électromagnétique plane dont le vecteur champ électrique est dans le plan d'incidence et dans un plan perpendiculaire au plan d'incidence.

2. Valeurs de l'angle d'incidence $\theta_{1}$ pour lesquelles il y a transfert d'énergie entre les milieux I et III. Tant que l'angle d'incidence $\theta_{1}$ dans le milieu I reste inférieur à l'angle de réflexion totale :

$$
\theta_{\mathrm{r}}=\arcsin \left(1 / n_{1}\right)
$$

à l'onde incidente correspondent à la fois une onde réfléchie et une onde réfractée.

Lorsque $\theta_{1}$ devient plus grand que l'angle de réflexion totale $\theta_{\mathrm{r}}$, il y a réflexion totale de l'onde incidente dans le milieu $I$ avec :

$$
\begin{gathered}
\sin \theta_{1}>1 / n_{1} \\
\cos \theta_{2}=-i\left(n_{1}^{2} \sin ^{2} \theta_{1}-1\right)^{1 / 2}
\end{gathered}
$$

puisque :

$$
n_{1} \sin \theta_{1}=\sin \theta_{2} .
$$

On a pris l'expression (3) de $\cos \theta_{2}$ avec le signe négatif car l'amplitude de l'onde électromagnétique dans la direction de propagation ne peut pas augmenter exponentiellement avec la distance de propagation.

De plus, l'équation suivante obtenue par application de la loi de Descartes :

$$
\cos ^{2} \theta_{3}=1-\frac{n_{1}^{2}}{n_{3}^{2}} \sin ^{2} \theta_{1}
$$

n'admet des racines réelles que si la condition :

$$
\sin \theta_{1}<n_{3} / n_{1}
$$

est vérifiée. Cette inégalité définit donc un angle limite $\theta_{1}$ d'incidence dans le milieu I :

$$
\theta_{1}=\arcsin \left(n_{3} / n_{1}\right)
$$

pour $n_{1}>n_{3}$. Si $n_{3}>n_{1}$, alors $\theta_{1}=\pi / 2$.

Dans le cas où $n_{1}>n_{3}$ et l'angle d'incidence $\theta_{1}$ est supérieur à $\theta_{1}$, il se produit la réflexion totale à la surface de séparation entre le milieu II et le milieu III, et comme résultat il n'y aura aucun transfert d'énergie du milieu I au milieu III. En résumé, il y a transfert d'énergie entre les milieux I et III pour des angles d'incidence $\theta_{1}$ satisfaisant aux conditions suivantes :

$$
\begin{array}{ccccc}
0<\theta_{1}<\theta_{1} & \text { avec } & \theta_{\mathrm{r}}<\theta_{1} & \text { si } & n_{1}>n_{3} \\
0<\theta_{1}<\pi / 2 & \text { avec } & \theta_{\mathrm{r}}<\pi / 2 & \text { si } & n_{3}>n_{1} .
\end{array}
$$

En faisant appel aux inégalités (2) et (6), on remarquera que l'on a toujours :

$$
\theta_{1}>\theta_{\mathrm{r}}
$$

3. Expression du transfert d'énergie rayonnante entre les milieux I et III. - Pour le corps noir, l'énergie rayonnante, dont le vecteur propagation est parallèle à une direction comprise à l'intérieur d'un cône élémentaire d'angle solide $\mathrm{d} \Omega=\sin \theta \mathrm{d} \theta \mathrm{d} \gamma$ et dont les longueurs d'onde émises sont comprises entre $\lambda$ et $\lambda+\mathrm{d} \lambda$, vaut :

$\mathrm{d} W=\frac{2 h c^{2}}{n^{2}} \cdot \frac{\mathrm{d} \lambda}{\lambda^{5}[\exp (h c / \lambda n k T)-1]} \sin \theta \mathrm{d} \theta \mathrm{d} \gamma$.

Donc le transfert d'énergie rayonnante du milieu I au milieu III vaut :

$$
\begin{gathered}
W_{\mathrm{I} \rightarrow \mathrm{III}}=\frac{4 \pi h c^{2}}{n_{1}^{2}}\left[\int_{0}^{\infty} \int_{0}^{\theta_{\mathrm{r}}}\left(\mathscr{J}_{\mathrm{I} \rightarrow \mathrm{III}}\right)_{\theta_{1}<\theta_{\mathrm{r}}} \cdot \frac{\sin \theta_{1} \cos \theta_{1} \mathrm{~d} \theta_{1} \mathrm{~d} \lambda}{\lambda^{5}\left[\exp \left(h c / \lambda n_{1} k T\right)-1\right]}\right. \\
\left.\quad+\int_{0}^{\infty} \int_{\theta_{\mathrm{r}}}^{\theta_{\mathrm{l}}}\left(\mathscr{J}_{\mathrm{I} \rightarrow \mathrm{III}}\right)_{\theta_{1}>\theta_{\mathrm{r}}} \cdot \frac{\sin \theta_{1} \cos \theta_{1} \mathrm{~d} \theta_{1} \mathrm{~d} \lambda}{\lambda^{5}\left[\exp \left(h c / \lambda n_{1} k T\right)-1\right]}\right] .
\end{gathered}
$$


Finalement, l'énergie échangée entre les milieux I et III vaut donc :

$$
W=W_{\mathrm{I} \rightarrow \mathrm{III}}-W_{\mathrm{III} \rightarrow \mathrm{I}} .
$$

4. Calcul du facteur de transmission pour les différents angles d'incidence $\theta_{1}$. - On va successivement calculer le facteur de transmission pour les deux cas suivants :

a) $0<\theta_{1}<\theta_{\mathrm{r}}$ (effet d'interférence);

b) $\theta_{\mathrm{r}}<\theta_{1}<\theta_{1}$ (effet tunnel).

Cas a). - Dans ce cas, le facteur de transmission est donné par la formule classique [2] :

$$
(\mathscr{J})_{\theta_{1}<\theta_{\mathrm{r}}}=\frac{n_{3} \cos \theta_{3}}{n_{1} \cos \theta_{1}} \cdot \frac{t_{12}^{2} t_{23}^{2}}{1+r_{12}^{2} r_{23}^{2}+2 r_{12} r_{23} \cos 2 \beta}
$$

où $t_{12}, t_{23}, r_{12}, r_{23}$, sont les coefficients de transmission et de réflexion de Fresnel pour les deux surfaces de séparation :

$$
\begin{aligned}
& t_{\perp}=\frac{2 n_{\mathrm{i}} \cos \theta_{\mathrm{i}}}{n_{\mathrm{i}} \cos \theta_{\mathrm{i}}+n_{\mathrm{t}} \cos \theta_{\mathrm{t}}} \\
& t_{l l}=\frac{2 n_{\mathrm{i}} \cos \theta_{\mathrm{i}}}{n_{\mathrm{t}} \cos \theta_{\mathrm{i}}+n_{\mathrm{i}} \cos \theta_{\mathrm{t}}}
\end{aligned}
$$

$$
\begin{array}{r}
r_{\perp}=\frac{n_{\mathrm{i}} \cos \theta_{\mathrm{i}}-n_{\mathrm{t}} \cos \theta_{\mathrm{t}}}{n_{\mathrm{i}} \cos \theta_{\mathrm{i}}+n_{\mathrm{t}} \cos \theta_{\mathrm{t}}} \\
r_{/ \prime}=\frac{n_{\mathrm{t}} \cos \theta_{\mathrm{i}}-n_{\mathrm{i}} \cos \theta_{\mathrm{t}}}{n_{\mathrm{t}} \cos \theta_{\mathrm{i}}+n_{\mathrm{i}} \cos \theta_{\mathrm{t}}}
\end{array}
$$

où $t_{\perp}, t_{\|}, r_{l l}, r_{\perp}$ ont leur signification classique, $n_{\mathrm{i}}$ est l'indice de réfraction du milieu incident et $n_{\mathrm{t}}$ celui du milieu réfractant, $\theta_{\mathrm{i}}$ et $\theta_{\mathrm{t}}$ les angles d'incidence et de réfraction.

On rappelle aussi l'expression de $\beta$ (différence de phase entre deux rayons réfléchis) :

$$
\beta=\frac{2 \pi}{\lambda} d \cos \theta_{2}
$$

où $d$ est l'épaisseur du milieu II d'indice $n_{2}=1$,

$\lambda$ est la longueur d'onde d'une radiation monochromatique.

Pour la suite, on pose :

$$
n_{1} \sin \theta_{1}=A \text {. }
$$

Tous calculs faits, le facteur de transmission pour une onde électromagnétique plane dont le vecteur champ électrique est dans le plan d'incidence vaut :

$$
\begin{array}{r}
\left(\mathscr{J}_{I I}\right)_{\theta_{1}<\theta_{\mathrm{r}}}=\frac{8\left(n_{1}^{2}-A^{2}\right)^{1 / 2}\left(1-A^{2}\right)\left(n_{3}^{2}-A^{2}\right)^{1 / 2}}{\left[n_{1}^{2}+1-\left(n_{1}^{2}+\frac{1}{n_{1}^{2}}\right) A^{2}\right]\left[n_{3}^{2}+1-\left(n_{3}^{2}+\frac{1}{n_{3}^{2}}\right) A^{2}\right]+4\left(n_{1}^{2}-A^{2}\right)^{1 / 2}\left(1-A^{2}\right)\left(n_{3}^{2}-A^{2}\right)^{1 / 2}} . \\
+\left[1-n_{1}^{2}+\left(n_{1}^{2}-\frac{1}{n_{1}^{2}}\right) A^{2}\right]\left[n_{3}^{2}-1+\left(\frac{1}{n_{3}^{2}}-n_{3}^{2}\right) A^{2}\right] \cos 2 \beta
\end{array}
$$

Pour une onde électromagnétique plane dont le vecteur électrique est dans un plan perpendiculaire au plan d'incidence, le facteur de transmission sera :

$$
\left(\mathscr{J}_{\perp}\right)_{\theta_{1}<\theta_{\mathrm{r}}}=\frac{8\left(n_{1}^{2}-A^{2}\right)^{1 / 2}\left(1-A^{2}\right)\left(n_{3}^{2}-A^{2}\right)^{1 / 2}}{\left(n_{1}^{2}+1-2 A^{2}\right)\left(n_{3}^{2}+1-2 A^{2}\right)+4\left(n_{1}^{2}-A^{2}\right)^{1 / 2}\left(1-A^{2}\right)\left(n_{3}^{2}-A^{2}\right)^{1 / 2}+\left(n_{1}^{2}-1\right)\left(1-n_{3}^{2}\right) \cos 2 \beta} .
$$

Pour une onde plane à l'incidence normale, on a : $A=0$ et on retrouve la formule connue suivante :

$$
\left(\mathscr{J}_{I I}\right)_{\theta_{1}<\theta_{\mathrm{r}}}=\left(\mathscr{J}_{\perp}\right)_{\theta_{1}<\theta_{\mathrm{r}}}=\frac{8 n_{1} n_{3}}{\left(1+n_{1}^{2}\right)\left(1+n_{3}^{2}\right)+4 n_{1} n_{3}-\left(n_{1}^{2}-1\right)\left(n_{3}^{2}-1\right) \cos 2 \beta} .
$$

CAS b). - Dans ce cas, la valeur de $\cos \theta_{2}$ est donnée par l'expression (3).

Les coefficients de transmission et de réflexion de Fresnel donnés par les expressions (13), (14), (15) et (16) deviennent maintenant complexes et prennent la forme suivante :

$$
\begin{gathered}
t_{\perp}=\tau_{\perp} \mathrm{e}^{i \chi_{\perp}} \\
t_{\| \prime}=\tau_{\| /} \mathrm{e}^{i \chi \| l} \\
r_{\perp}=\rho_{\perp} \mathrm{e}^{i \psi_{\perp}}=\mathrm{e}^{i \psi_{\perp}} \\
r_{\|}=\rho_{\| /} \mathrm{e}^{i \psi \|}=\mathrm{e}^{i \psi \|}
\end{gathered}
$$

puisque, dans ce cas, $\rho_{\perp}=\rho_{I I}=1$.
Les expressions précédentes prennent une forme explicite en les exprimant en fonction des constantes optiques et des paramètres convenables.

L'expression (17) de $\beta$ devient :

$$
\beta=-i \frac{2 \pi}{\lambda} d\left(n_{1}^{2} \sin ^{2} \theta_{1}-1\right)^{1 / 2}=i b .
$$

Le facteur de transmission prend la forme suivante :

$$
(\mathscr{J})_{\theta_{1}>\theta_{\mathrm{r}}}=\frac{n_{3} \cos \theta_{3}}{n_{1} \cos \theta_{1}} \cdot \frac{\tau_{12}^{2} \tau_{23}^{2}}{2 \cos \left(\psi_{12}+\psi_{23}\right)+2 \cosh 2 b} \text {. }
$$


Les facteurs de transmission $\left(\mathscr{J}_{I I}\right)_{\theta_{1}>\theta_{\mathrm{r}}}$ et $\left(\mathscr{J}_{\perp}\right)_{\theta_{1}>\theta_{\mathrm{r}}}$ prennent respectivement les expressions suivantes :

$$
\begin{array}{r}
\left(\mathscr{J}_{I I}\right)_{\theta_{1}>\theta_{\mathrm{r}}}=\frac{8\left(n_{1}^{2}-A^{2}\right)^{1 / 2}\left(1-A^{2}\right)\left(n_{3}^{2}-A^{2}\right)^{1 / 2}}{\left[1+n_{1}^{2}-\left(n_{1}^{2}+\frac{1}{n_{1}^{2}}\right) A^{2}\right] \cdot\left[1+n_{3}^{2}-\left(n_{3}^{2}+\frac{1}{n_{3}^{2}}\right) A^{2}\right]+4\left(n_{1}^{2}-A^{2}\right)^{1 / 2}\left(1-A^{2}\right)\left(n_{3}^{2}-A^{2}\right)^{1 / 2}} \\
\quad+\left[1-n_{1}^{2}+\left(n_{1}^{2}-\frac{1}{n_{1}^{2}}\right) A^{2}\right]\left[n_{3}^{2}-1+\left(\frac{1}{n_{3}^{2}}-n_{3}^{2}\right) A^{2}\right] \cosh 2 b
\end{array}
$$

$$
\left(\mathscr{J}_{\perp}\right)_{\theta_{1}>\theta_{\mathrm{r}}}=\frac{8\left(n_{1}^{2}-A^{2}\right)^{1 / 2}\left(1-A^{2}\right)\left(n_{3}^{2}-A^{2}\right)^{1 / 2}}{\left[n_{1}^{2}+1-2 A^{2}\right]\left[n_{3}^{2}+1-2 A^{2}\right]+4\left(n_{1}^{2}-A^{2}\right)^{1 / 2}\left(1-A^{2}\right)\left(n_{3}^{2}-A^{2}\right)^{1 / 2}+\left(n_{1}^{2}-1\right)\left(1-n_{3}^{2}\right) \cosh 2 b} .
$$

5. Energie échangée entre les deux diélectriques. On obtient les facteurs de transmission pour l'énergie rayonnante :

$$
\left(\mathscr{J}_{\mathrm{I} \rightarrow \mathrm{III}}\right)_{\theta_{1}<\theta_{\mathrm{r}}} \text { et } \quad\left(\mathscr{J}_{\mathrm{I} \rightarrow \mathrm{III}}\right)_{\theta_{1}>\theta_{\mathrm{r}}}
$$

en substituant les expressions (19), $(20),(28)$ et $(29)$ dans (1).

Une fois que les expressions (30) sont substituées dans (10), on obtient finalement l'expression de l'énergie transférée du milieu I au milieu III et inversement.

On remarquera l'extrême complexité de l'expression (11) donnant l'énergie échangée entre les deux milieux diélectriques I et III.

On s'est borné à des solutions numériques de l'expression (10) pour différentes valeurs des indices de réfraction et différentes températures.

Il est fort connu que le facteur de transmission d'une onde monochromatique, pour le cas indiqué sur la figure 1, est une fonction périodique de l'épaisseur $d$ du milieu II : les extremums du facteur de transmission se produisant pour :

$$
\begin{array}{lll}
d=m \lambda_{0} / 4 n_{2} \cos \theta_{2} \quad \text { avec } \quad & \theta_{1}<\theta_{\mathrm{r}} \\
& (m=1,2,3 \ldots)
\end{array}
$$

on aura un maximum ou un minimum selon que l'expression suivante :

$(-1)^{m} r_{12} r_{23}\left[1+r_{12}^{2} r_{23}^{2}-r_{12}^{2}-r_{23}^{2}\right] \quad(m=1,2 \ldots)$ est positive ou négative [3].

Il va de soi que, lorsque l'épaisseur $d$ du milieu II est égale à zéro, le facteur de transmission sera maximum.

Dans le cas présent, la source rayonnante n'est pas monochromatique, bien que plus de $95 \%$ de l'énergie rayonnante soit transportée par les radiations dont les longueurs d'onde, à une température donnée, sont comprises entre $\lambda_{\mathrm{m}} / 2$ et $5 \lambda_{\mathrm{m}}$, où $\lambda_{\mathrm{m}}$ est tirée de la loi de déplacement de Wien.
On a représenté sur la figure 2 l'énergie échangée pour $\theta_{1}<\theta_{\mathrm{r}}$ en fonction de $d /\left(\lambda_{\max }\right)_{T_{3}}$ où $\left(\lambda_{\max }\right)_{T_{3}}$ est calculé à la température $T_{3}$ et dans le vide; on

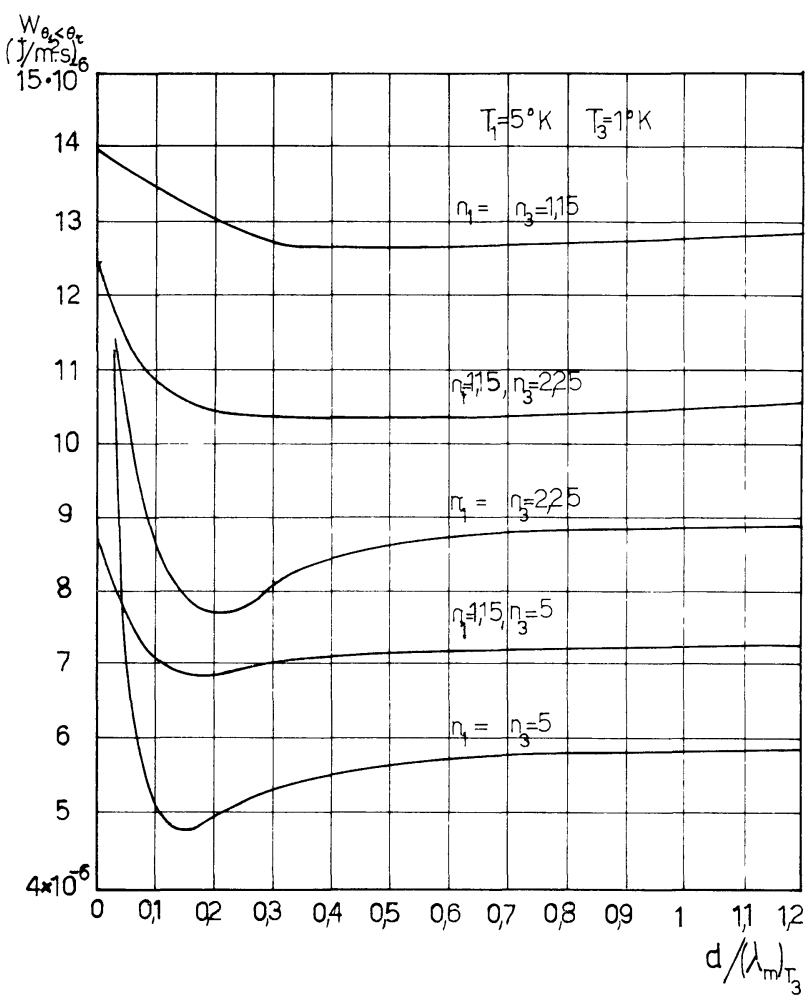

FIG. 2. - Échange d'énergie entre les deux diélectriques par transmission, compte tenu de l'effet d'interférence.

remarque que le minimum d'énergie échangée a lieu pour des valeurs du rapport $d /\left(\lambda_{\max }\right)_{T_{3}}$ d'autant plus petites que les indices de réfraction des milieux I et III sont plus élevés. De même, si l'on fait varier la température du milieu I, le minimum d'énergie échangée se produit pour des valeurs de $d /\left(\lambda_{\max }\right)_{T_{3}}$ d'autant plus petites que la température $T_{1}$ est plus élevée, comme 


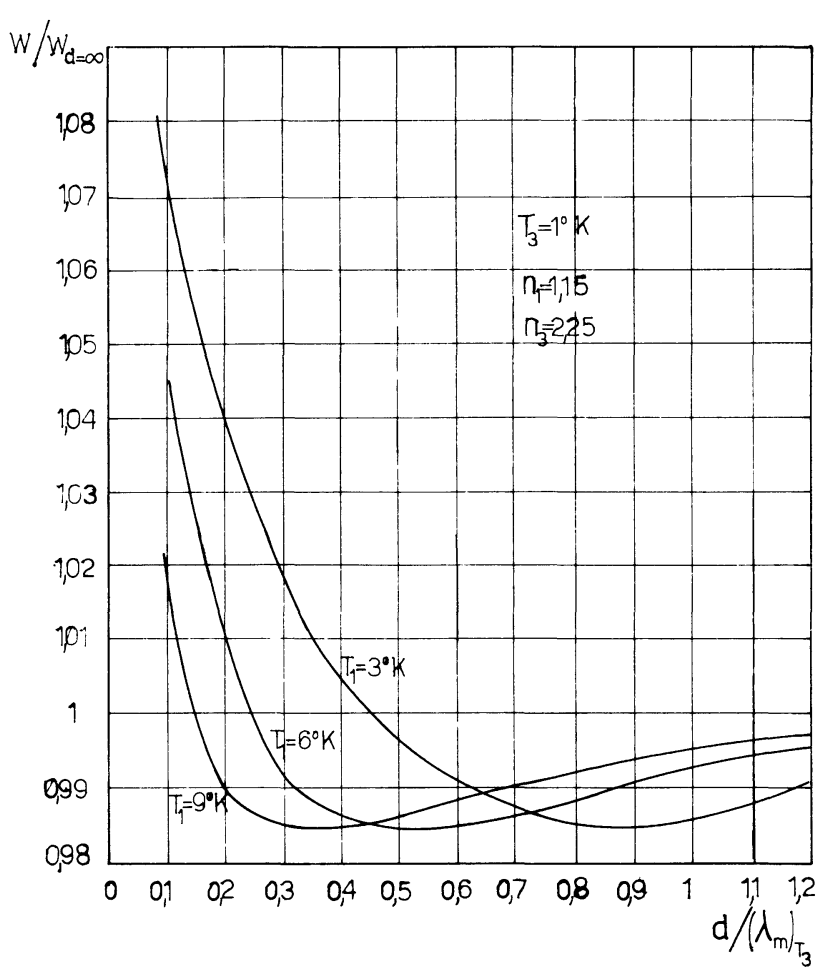

FIG. 3. - Influence de la température sur l'échange d'énergie par transmission, compte tenu de l'effet d'interférence.

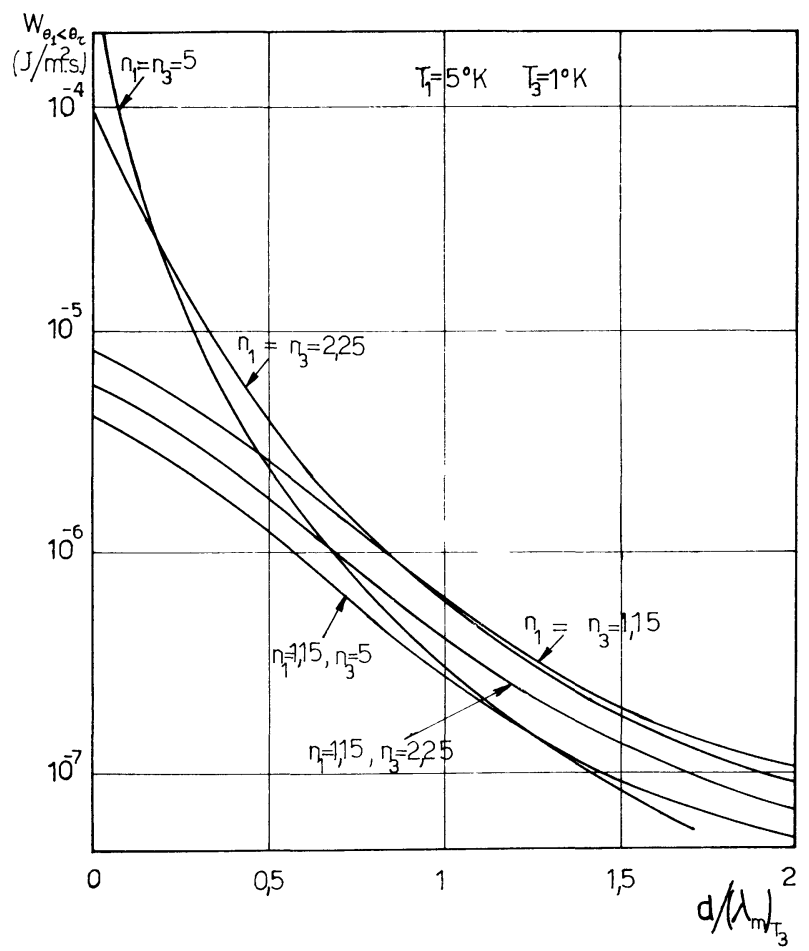

FIG. 4. - Échange d'énergie entre les deux diélectriques par effet tunnel. l'indique la figure 3 où l'énergie échangée est rapportée à celle qu'on aurait pour $d=\infty$.

L'influence des indices de réfraction et de la température sur l'énergie échangée s'explique aisément si l'on tient compte du fait que le produit $n \lambda$ est une constante pour des milieux ayant un indice différent et $\lambda$ étant la longueur d'onde prise par une même radiation dans ces différents milieux.

Donc les valeurs des longueurs d'onde qui prédominent dans le rayonnement thermique diminuent lorsque l'indice de réfraction du milieu augmente; de même quand la température augmente, $\lambda_{\max }$ devient plus petit, si bien que le minimum pour le facteur de transmission se produira pour des valeurs de la distance $d$ plus petites, fait bien connu en optique et en électromagnétisme. Sur la figure 4 , on a représenté l'énergie échangée par effet tunnel, c'est-à-dire pour $\theta_{1}>\theta_{\mathrm{r}}$ et sur la figure 5 on montre l'influence de la température sur cette même énergie.

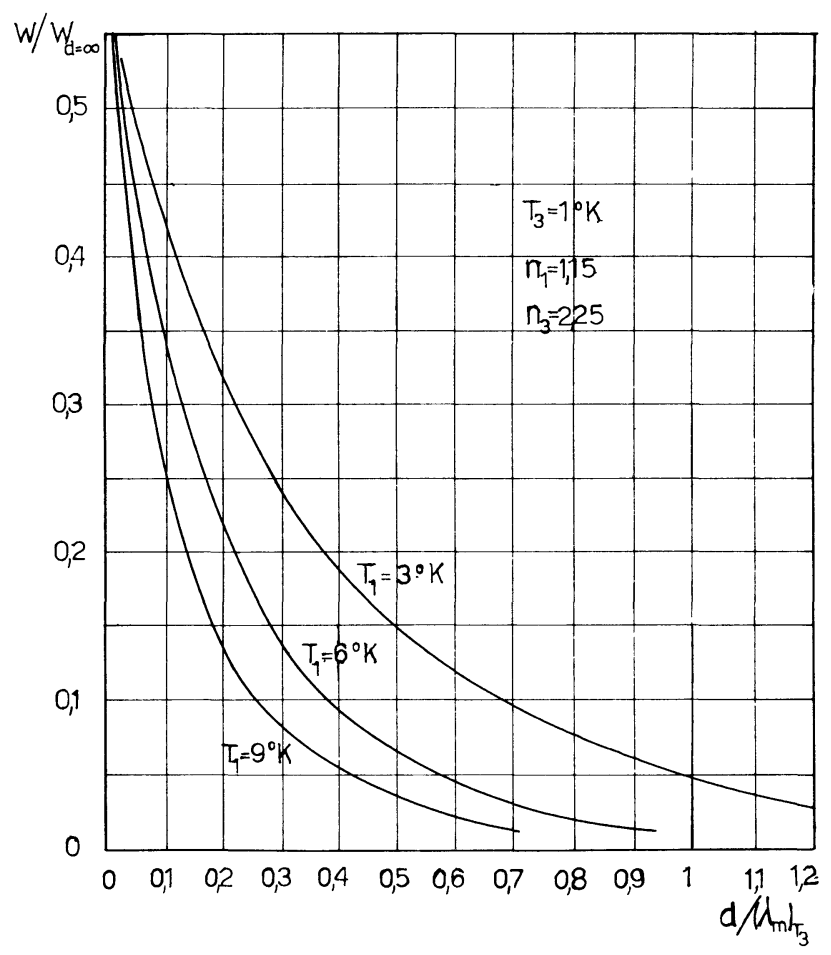

FIG. 5. - Influence de la température sur l'échange d'énergie par effet tunnel.

Il est fort connu que l'amplitude de l'onde évanescente décroît d'autant plus rapidement que sa longueur d'onde est plus petite et donc dans les figures 4 et 5 l'atténuation de l'énergie échangée par effet tunnel est d'autant plus rapide que les indices de réfraction, ainsi que la température, sont plus élevés.

L'allure de l'énergie totale échangée, somme de 
l'énergie échangée par transmission $\left(\theta_{\mathbf{1}}<\theta_{\mathrm{r}}\right)$ et par effet tunnel $\left(\theta_{1}>\theta_{\mathrm{r}}\right)$ en fonction de $d /\left(\lambda_{\max }\right)_{T_{3}}$, est donnée sur les figures 6 et 7 .

On remarquera que la contribution par effet tunnel est telle que le minimum d'énergie transmise pour $\theta_{1}<\theta_{\mathrm{r}}$ n'apparaît plus dans l'énergie totale échangée,

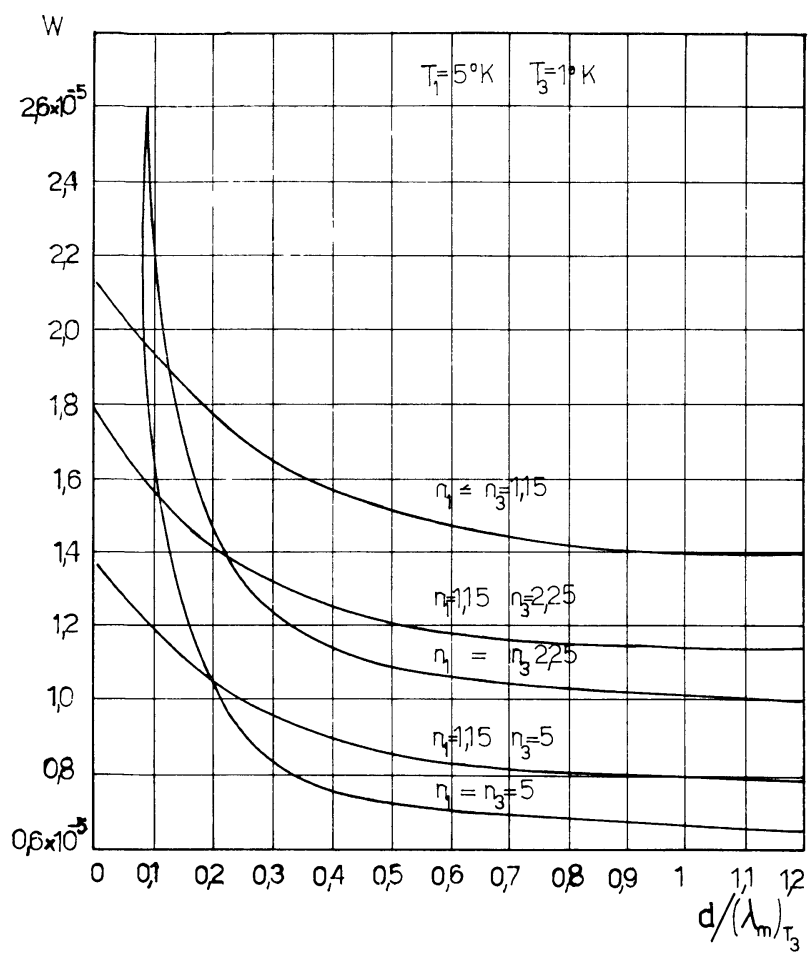

FIG. 6. - Échange total d'énergie entre les deux diélectriques. qui ne présente pas de minimum. On voit que l'atténuation de l'énergie totale échangée est d'autant plus rapide que les indices de réfraction et la température sont élevés. A partir de $d /\left(\lambda_{\max }\right)_{T_{3}}=1$, la valeur de l'énergie totale échangée coïncide avec celle donnée par la solution classique.

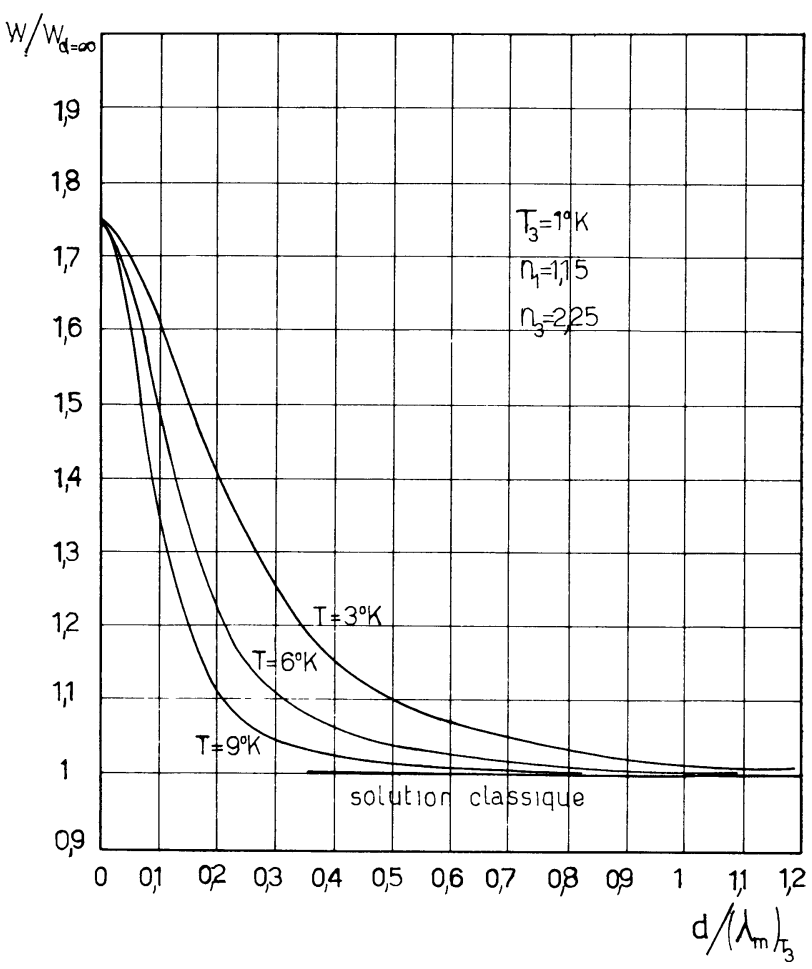

FIG. 7. - Influence de la température sur l'échange d'énergie totale.

\section{BIBLIOGRAPHIE}

[1] Stern, "Solid State Physics », Academic Press, 1963, 15,323 .

[2] BORN et WOLF, "Principles of Optics ", Pergamon Press, 1965, p. 62.

[3] BORN et WOLF, op. cit., p. 63. 